\title{
Linking Structure to Function: The Search for Active Sites in Non-Platinum Group Metal Oxygen Reduction Reaction Catalysts
}

\author{
Edward F. Holby \\ Sigma Division, Los Alamos National Laboratory, Los Alamos, NM 87545 \\ Piotr Zelenay \\ Materials Physics and Applications Division, Los Alamos National Laboratory, Los \\ Alamos, NM 87545
}

\begin{abstract}
Atomic-scale structures of oxygen reduction reaction (ORR) active sites in non-platinum group metal (non-PGM) catalysts, made from pyrolysis of carbon, nitrogen, and transition-metal (TM) precursors have been the subject of continuing discussion in the fuel cell electrocatalysis research community. Quantum chemical modeling is one path forward for understanding of these materials and how they catalyze the ORR. We here demonstrate through literature examples of how such modeling can be used to better understand non-PGM ORR active site relative stability and activity and how such efforts can also aid in the interpretationof experimental signatures produced by these materials.
\end{abstract}

\section{TOC Figure:}

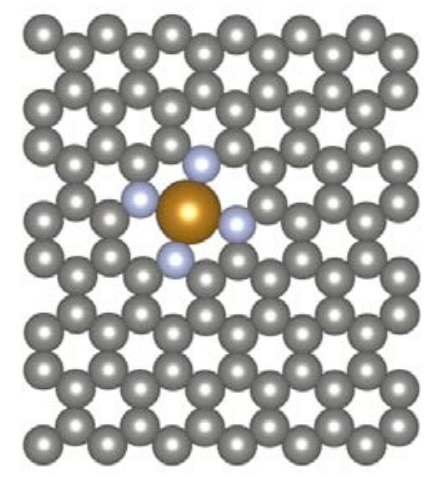

Keywords:non-PGM catalysts; oxygen reduction reaction; ORR; DFT modeling; fuel cells 


\section{Background}

The use of non-platinumgroup metal (non-PGM) catalysts for the oxygen reduction reaction (ORR) that occurs at the cathode of proton exchange fuel cells (PEFCs) is not tied to the availability of a scarce commodity as it is for Pt-based catalysts. NonPGM catalyst materials, based on precursors of Earth-abundant elements of carbon (C), nitrogen (N), and transition metals (TMs), have undergone several dramatic shifts in how they are synthesized, leading to drastically improved ORR activity and durability under fuel cell conditions[1-7]. Despite the improvements, these two catalyst properties, as well as cost of synthesis and reaction selectivity, need even further optimization for non-PGM catalysts to be commercially relevant replacements for Pt-based PEFC cathode materials.

At the heart of improving non-PGM catalysts lies the mystery of the atomicscale structures of active sites. Due to the pyrolysis of precursor materials in stateof-the-art non-PGM materials synthesis, a wide variety of structures are formed and understanding which component of the material is responsible for ORR activity is still debated. There is still much discussion, in particular, regarding the role of transition metal precursors and whether they are a central component of the activesite structure or whether they are simply required to aid in templating/catalyzing formation of $\mathrm{C}-\mathrm{N}$ active sites.

This gap in knowledge regarding the fundamental nature of the active site is problematic for a rational approach to catalyst improvement. ORR activity is a function of both active-site density within the material and the turnoverfrequency (TOF) of the sites[4]. Ideally, a material would have a high density of high-TOF sites. Exposure of materials to electrochemical environments may lead to corrosive attack or poisoning of active sites leading to a loss of catalytic activity. As such,active sites should be resistant to corrosion and poisoning in order to maintain high activity under operating conditions for long periods. Knowing how atomic-scale structure couples to both activity and durability could help to guide synthesis to achieve this high density of very active and corrosion-resistant sites.

The last decade has seen acceleration of non-PGM development, as well as the application of quantum chemical calculations for understanding and improving electrocatalysts[8-14]. These calculations can provide valuable insight into electrochemical reaction mechanisms and have matured to the point that prediction of ORR activity as a function of atomic-scale activesite structure is now routine in the literature. In addition to forecasting catalyst activity, these techniques can also be utilized to understand the active-site thermodynamics, which can in turn aid in understanding the probability of given structures being formed and how stable they may be relative to other structures. As the field advances, predictions of mechanismspecific corrosion properties as a function of structure are likely not far off. Quantum chemical calculations can also be useful for trying to de-convolute complicated experimental signatures in pyrolyzed materials and facilitate understanding of the experimental output.

This manuscript contains examples how quantum chemical modeling has been applied to the understanding of non-PGM catalyst materials, with particular attention to linking atomic-scale structures to calculated formation energies, ORR 
activities in acidic environments, and experimental signatures. It is not meant as an exhaustive review of available modeling literature but to selectively utilize key publications in order to highlight specific points regarding the importance of atomic-scale structure. It is our hope that this discussion will spur future investigators to consider the use of quantum chemical modeling in their research and to serve as a starting point for such efforts.

\subsection{Density Functional Theory}

Density functional theory (DFT), when coupled with thermochemical models to form a quantum chemical model, makes for a powerful computational tool for better understanding catalyst properties, including relative stability[15-22], ORR activity[13, 16, 20, 22-29], and selectivity[22, 26, 30,31]. DFT capabilities are reflected by its increasing use in the field of catalysis. A full description of DFT is beyond the scope of this work but, in brief, DFT solves an approximation of the many-body (electron) Schrödinger equation for an initial guess of atomic structure and returns calculated locally relaxed ground state atomic and electronic structures and relative structure internal energies at $0 \mathrm{~K}$. DFT is well suited to calculations of $\sim 100$ atoms and can capture much of the physics important for understanding nonPGM materials at the atomic scale. In particular, formation energies and binding energies are important quantities that can be calculated via DFT.These values can be otherwise difficult to determine accurately at the atomic level for structures on the $\sim 100$ atom size scale. Mass-transport contributions to ORR overpotential require larger time- and length-scale approaches[32] and so the relative activities from DFT calculations arelimited to the electrode kinetics, represented by the kinetic regionin catalyst polarization plots. Despite this limitation, the approach is still invaluable for understanding ORR at the atomic scale and comparing varied atomic-scale structures.

In the approaches discussed below, DFT calculatedinternal energies are utilized as input for thermochemical models for relative stability and activity. In addition, DFT can be used to obtain vibrational data for calculating zero-point energy (ZPE) and vibrational contributions to entropy. Addition of a solvent and its energetic effects is also possible, though $a b$ intio molecular dynamics or addition of a solvation field. Other approaches can add in the effect of double-layer structures generated due to charged surfaces. These approaches attempt to give a more accurate representation of system free energies under in-situ conditions. A number of authors have shown, however, that trends in activity can be identified, in most cases without these computationally expensive additions[8, 12, 33].Further refinements of the thermochemical models and of the DFT values used as input for these models is the focus of many current research efforts.

\section{Linking Synthesis Conditions and Structures}

Using DFT-calculated energy values for given atomic-scale structures, it is possible to determine formation energies for active site structuresversus chosen reference states for each of the considered chemical species. These formation energies can be 
used to compare the relative stability of active site structures.For instance, if we consider the $\mathrm{FeN}_{4}$ structure shown in Figure 1, there are three chemical species, $\mathrm{C}$, $\mathrm{N}$, and Fe. One choice of reference states is graphene for carbon, molecular $\mathrm{N}_{2}$ for nitrogen, and bulk body-centered cubic (BCC) Fe for iron. Each of these reference states has a (per atom) reference state energy that can be calculated via DFT $\left(E_{x}\right.$, where $x$ is the considered species). These reference states represent the chosen zero point of chemical potential for each of the species. DFT calculation of the FeN 4 structure energy can then be compared to the same number of atoms of a given species at their reference state to give a relative formation energy, $E_{\text {formation }}$, given in Equation [1]:

$E_{\text {formation }}=E_{\text {atoms in structure }}-E_{\text {atoms in reference state }}$

For the specific case of the FeN $\mathrm{N}_{4}$ structurein Figure 1, where the Fe occupies a C bivacancy, the $\mathrm{N}$ replace 4 neighboring $\mathrm{C}$ atoms, and the initial, defect-free graphene structure contains $96 \mathrm{C}$ atoms, the relative formation energy can be calculated as:

$$
E_{\text {formation }, \mathrm{FeN}_{4}}=E_{\mathrm{FeN}_{4}}+6 E_{\mathrm{C}_{3} \text { graphene }}-96 E_{\mathrm{C}_{3} \text { graphene }}-2 E_{\mathrm{N}_{2}}-E_{\mathrm{Fe}, \mathrm{bcc}}
$$

In this example, $6 \mathrm{C}$ atoms are removed from the 96 atom graphene structure to host the Fe and $\mathrm{N}$ ( 2 for the Fe and 4 that are replaced by $\mathrm{N}$ atoms) and placed at their reference-state energy. The initial state is defined as the defected-free 96 atom graphene sheet, $2 \mathrm{~N}_{2}$ molecules ( $4 \mathrm{~N}$ atoms), and $1 \mathrm{Fe}$ atom. If formation energy calculated in this manner is negative, then formation of the defect site is thermodynamically stable versus the choice of reference states. By comparing formation energies of different structures, the relative stability is obtained. Assuming a thermodynamic distribution of sites leads to the conclusion that the lower (more negative) the formation energy, the higher the concentration of particular sites. In this way, DFT can be input into a thermochemical formation energy model to compare the likelihood of given sites being present. This approach is agnostic as to the formation pathway and thus ignores any potential kinetic barriers that must be overcome during the synthesis process. Such kinetic barriers are possibly why relatively high pyrolysis temperatures are required in order to achieve highly active catalysts [4].

Through the use of this DFT-based relative stability formalism, the authors have developed three structural motifs governing Fe-N defects in graphene hosts (in the form of either a graphene nanoribbon or a graphene "bulk" sheet)that capturecalculated trends in active-site relative formation energies[17-19]:

1) Fe substitutional atoms are significantly stabilized by N-coordination. This appears to be true for both three-fold coordination (Fe replacing a single $\mathrm{C}$ atom) as well as for four-fold coordination (Fe replacing $2 \mathrm{C}$ atoms). 
2) Fe-N defects are more stable at the nanoribbon edges $v s$. central positions. The formation energies at central positions of nanoribbons are slightly lower than those calculated in bulk graphene. Comparing defect formation energies at edges $v s$. bulk show that for $\mathrm{FeN}_{3}$ structures, the edge site is $\sim 2.4 \mathrm{eV}$ more stable than bulk (edge structure shown in Figure 2) and that for the $\mathrm{FeN}_{4}$ structure, the edge site is $\sim 1.6 \mathrm{eV}$ more stable than bulk for the chosen reference states. This difference is likely due to the increased perturbation associated with an $\mathrm{Fe}$ at a $\mathrm{C}$ monovacancy $\left(\mathrm{FeN}_{3}\right)$ (also considered by Kabir $e t$ $a l$. [20]) and the increased ability of the edge to relax this larger perturbation. A similar stabilization effect has been reported for Fe-N defects near pore structures[16,27], again, likely due to the ability of the local C structure to relax near open edges.

3) There is a thermodynamic driving force for edge $\mathrm{FeN}_{\mathrm{x}}$ defects to form small clusters[19]. For the $\mathrm{FeN}_{3}$, there is a particularly strong thermodynamic difference, when Fe atoms are at second nearest neighbor positions with a bridging/shared $\mathrm{N}$. The driving force is less strong for the in-plane $\mathrm{FeN}_{4}$ structures. Such closely clustered metal sites are likely to have dramatically different ORR behavior compared to single metal atom sites. Multi-metal atom sites are also more likely to experience significant kinetic formation limitations due to the increased structural complexity and the need to have multiple local metal atoms present to form such structures. For Fe and Co, a thermodynamic alloying effect has been demonstrated indicating that combination of $\mathrm{Fe}$ and $\mathrm{Co}$ is energetically favorable versus formation of segregated structures[24].

Deviations from chosen reference states can be treated as a change in the chemical potential of a given species and enteredinto Equations [1] and [2] via altered $E_{x}$ values. The chosen reference state can act as a zero(reference) value for chemical potential and the above formation energy model can be modified to include shifts in chemical potential from this state. As an example, the $\mathrm{N}_{2}$ molecule reference state could be altered by temperature-dependent contributions to more accurately capture temperature dependencies on formation, changes in $\mathrm{N}_{2}$ partial pressure to capture environmental effects,or use of a higher energy $\mathrm{NH}_{3}$ reference state to simulate more directly certain pyrolysis approaches. These changes can be included by a shift in the reference state for $\mathrm{N}$ species. (Quantitative valuesfor such energy shifts are often available via tabulated chemical data or via thermodynamic calculation, which can give a range of relevant chemical potential shifts or be utilized to refine reference state values. Such shifts can also be utilized to capture thermodynamic properties of states beyond calculated internal energies.) Changes in reference state can affect the calculated relative stability of structures with differing amounts of a given species, such as $\mathrm{N}$. By including this as a shift in chemical potential, Holby et al.determined that there are a variety of stable $\mathrm{Fe}-\mathrm{N}-\mathrm{C}$ structures possible in a zig-zag nanoribbon host C-structure (Figure 3 ) and that which structure has the highest relative stability (represented by the lowest formation energy at a given chemical potential) is a function of both $\mathrm{N}$ and Fe 
chemical potentials[19]. This indicates that choice of precursor and processing conditions can alter the thermodynamically most stable Fe-N-C structure, allowing for some tunability of synthesized active sites.

DFT-based formation energies also enable thermodynamic comparisons ofthe predominance ofactive-site structures. For instance, structural motif in item 2 above, points toward similar structures being more stable at graphene edges $v s$. bulk.With calculated formation energies, simple assumptions, and application of a Gibbs Measure to describe distribution probability, an estimation of the relative ratio of edge-to-bulk $\mathrm{FeN}_{\mathrm{X}}$ structures can be determined. If we assume that there are only two states (edge and bulk) and the probability, $P$, of each state is given by a canonical partitioning weighted by the concentration of host sites ( $C_{\text {edge }}$ for edge $\mathrm{C}$ and $c_{\text {bulk }}$ for bulk C), then, the ratio of edge to bulk sites should be given by Equation [3]:

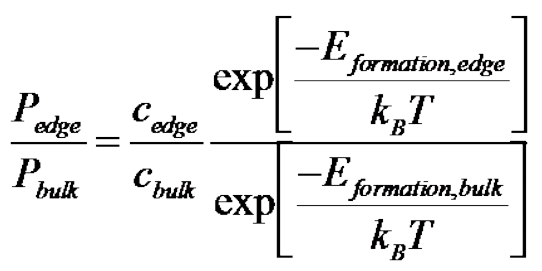

In this equation, $E_{\text {formation }}$ represents the relative calculated formation energies, $k_{B}$, is the Boltzmann constant, and $T$ is the temperature in Kelvin.If we consider the $\mathrm{FeN}_{3}$ case and use bulk formation energy as a reference value of zero and a formation temperature, $T$, of $1173 \mathrm{~K}\left(900^{\circ} \mathrm{C}\right.$, a common pyrolysis temperature), for there to be an even distribution of edge and bulk sites, the ratio of bulk to edge host sites $\left(c_{\text {bulk }} / c_{\text {edge }}\right)$ must be of the order $10^{10}$. For $\mathrm{FeN}_{4}$, the ratio is of the order $10^{6}$, still a large number. Even for this lower value, if we assume a zig-zag ribbon geometry composed of only edge and bulk sites ( 2 edge sites and $l$ bulk sites where $l$ is given by the ribbon width, $d$, and linear C density, $r$, such that $l \sim d \times r$ ), to match such a ratio, the ribbon would need to be hundreds of micronsthick $(d \sim 0.5 \mathrm{~mm}$ for $r=1 \mathrm{C}$ / $\sim 1 \AA$ A)to satisfy such a site ratio. Such a large width is unrealistic for a high surface area carbon with typical lengths on the nanometer scale, thus indicating the likely predominance of edge structures (assuming a thermodynamic distribution). Edge sites are expected to have a kinetic advantage in formation as well, since they are more accessible to $\mathrm{Fe}$ and $\mathrm{N}$ reactants during synthesis. This simple thermodynamic model, enabled by DFT calculations, highlights the dramatic difference in site concentrations required for an equal thermodynamic distribution between states, heavily favoring defect formation at edges. It also demonstrates the type of datadriven considerations that will aid in understanding the atomic nature of non-PGM ORR active sites.

Another useful approach for considering structural stability is discussed by Kwak et al.[20],who utilized a binary alloy approach for understanding relative stability of C-N structures in both bulk and zig-zag nanoribbon structures. There has 
been an ongoing debate regarding the effect of $\mathrm{N}$ concentration and speciation of $\mathrm{C}-\mathrm{N}$ structures on ORR catalyst activity, particularly in the context of metal-free ORR active sites.Through DFT-calculated formation energies of over 700 bulk N-doped graphene structures and over 100 edge $\mathrm{N}$-doped graphene nanoribbon structures, Kwak et al.[20]developed a convex hull model, showing relative thermodynamic stability of phases as a function of N-doping concentration. For bulk N-doped graphene structures, a specific ordering of $\mathrm{N}$ at a fractional concentration of 27.8 at. $\% \mathrm{~N}$ was found to be a formation energy minima and concentration values above this are likely to phase-segregate between this $27.8 \mathrm{at} . \% \mathrm{~N}$ phase and the chosen end-point for more highly N-doped systems (Figure 4). At lower concentrations, a variety of $\mathrm{C}-\mathrm{N}$ phases were found to be on the calculated convex hull including a 5.5 at. $\% \mathrm{~N}$ phase, 11.1 at. $\% \mathrm{~N}$ phase, 16.7 at. $\% \mathrm{~N}$ phase, and a 22.2 at. $\% \mathrm{~N}$ phase. Thus, the predicted thermodynamic phase is strongly dependent upon the $\mathrm{N}$ concentration. $\mathrm{N}$ concentrations between these phases should phase-segregate to the two neighboring phases following a lever rule, however, due to the coarse graining of $\mathrm{N}$ concentrations required by finite sized calculation cells, it is possible that other intermediate phases exist along the convex hull. Based on literature values of intercalated $\mathrm{N}$ concentrations, two of thedetermined phases are of particular interest, one occurring at 5.5 at.\% $\mathrm{N}$ and one at 11.1 at.\% $\mathrm{N}$. These phases show a lack of N-clustering with the closest $\mathrm{N}-\mathrm{N}$ distance being at third nearest-neighbor positions (11.1 at.\% N). This finding has implications regarding the nature of metalfree (only $\mathrm{C}$ - and $\mathrm{N}$-based) bulk ORR active-site structures for materials since this prediction of separated $\mathrm{N}$ is likely to affect $\mathrm{O}_{2}$ binding energies as discussed in the activity section.

For the considered zig-zag nanoribbon structure in Kwak et al.[20], the minimum formation-energy phase occurs for $\mathrm{N}$ occupying all of the edge positions of the ribbon. For their chosen ribbon, this is a 33.3 at.\% $\mathrm{N}$ doping but further calculations are required to determine if this edge behavior dominates for thickerribbons. If the trend of $\mathrm{N}$ replacing only edge $\mathrm{C}$ atoms would hold, then this would occur at $\mathrm{N}$ doping concentrations well below the determined 33.3 at.\% level reported in this study for thicker ribbons (with more non-edge $\mathrm{C}$ ). For instance, edge occupation of an8 $\mathrm{C}$ pair thick ribbon, an edge-only $\mathrm{N}$ occupation structure would be 12.5 at.\% N. This finding also has implications for the nature of ORR active-site structures, in particular that edge occupation by $\mathrm{N}$ is strongly favored $v s$. more bulk-like positions, an effect that is likely to enhance formation probability of edge-based $\mathrm{N}$-containing structures. Other researchers have consideredactive-site structures with $\mathrm{N}$ at positions other than the position predicted by this work. Such states are meta-stable to the structures reported by Kwak et al.[20]and thus are thermodynamically less likely to be formed.

Convex hull calculations as well as formation energy values as a function of chemical potential based on DFT calculations give insight into which structures are thermodynamically favored to form as a function of varied environments. As such, these studies provide useful starting points for determining which atomic-scale structures should be considered for ORR active-site candidates. High-throughput calculations of formation energies can be used to down-select structures for ORR activity calculation and may aid in determining new structures not previously 
considered for active-site structures. An important distinction must be made, however, between calculated relative stability between phases and the durability of active sites. While the relative stability can dictate the thermodynamic probability of given structures occurring in the synthesized material, the durability is dependent upon the specific corrosion mechanism that leads to loss of active-site structures in the relevant environment. For instance, edge sites have been shown to have a significantly lower formation energy than their bulk analogs. Edges, however, may be more susceptible to corrosion of the $C$ support and thus edge sites may be more prone to corrosion and deactivation. More research into the corrosion mechanisms behind theloss of ORR active sites and of the effects of active site structure on durability [34] is required to couple DFT-based studies to non-PGM catalyst longterm durability.

\section{Linking ORR Activity and Structures}

One of the keys to improving non-PGM catalysts is increasing the site density of the most ORR-active structures since total activity is a function of both number of active sites and the turnoverfrequency (TOF) of those sites. Understanding what activesite structures lead to high ORR activity and high TOF would greatly aid in the pursuit of creation of more active materials, providing target structures for synthesis. In this section, we will discuss how DFT modeling has been applied to understand the ORR reaction in acidic environments and how active-site structure (particularly local C structure, as well asN-doping and speciation) couples to activity.

For over a decade, DFT studies of ORR activity have been used to help guide synthesis of catalyst materials. This is due largely to the success of the computational hydrogen electrode (CHE) thermochemical model, described by Norskov, et al.[8] and other models that produce a DFT-accessible descriptor of ORR activity. In this methodology, DFT is used to calculate binding energies of ORR intermediates on active-site structures. These binding energies, as well as carefullychosen reference states,give energy values to chosen sub-steps of the ORR reaction (for example, those given by Equation[4a-e]), as well as the system energy dependence on potential. Combining these system energies provides a calculated thermodynamic reaction pathway as a function of potential, which is added postcalculation as part of the CHE thermochemical model. This approach enables calculation of the thermodynamic limiting potential, $U_{l}$, above which there is a thermodynamic barrier between two or more states. In addition, the potentialdetermining step, the reaction that sets the limiting potential, can also be calculated in this formalism. The value of $U_{l}$ can serve as a DFT-accessible descriptor of ORR activity and when coupled with the potential-determining step is useful for uncovering trends in activity as a function of atomic-scale structure. Examples of these quantities and their more detailed description are given in the next section.

While ignoring kinetic barriersbetween thermodynamic statesand non-local electronic / chemical effects, this approach enables a direct comparison of predicted ORR activities as a function of active-site atomic-scale structure for a given reaction pathway. Additionally, different reaction pathways can be tested to determine if, for 
example, an associative vs. dissociative mechanism is likely to occurin the ORR or if $\mathrm{H}_{2} \mathrm{O}_{2}$ is thermodynamically likely to be formed, which would affect catalyst selectivity. This approach has been widely utilized in understanding how metal and metal alloy surfaces can be tuned to improve ORR activity but researchers have also utilized this method for understanding the activity of proposed non-PGM active-site structures. This approach is particularly useful for considering the role of transition metals in the active-site structure. An example of how this approach can be utilized, followed byliterature examples of ORR activity calculations for both metal-bearing and metal-free structures, are given in this section.

\section{CHE Methodology Example}

The CHE methodology enables charge-neutral DFT calculations to be used to approximate the effects of electric potential on electrochemical reaction pathways. Detailed discussions are available in references[8, 12, 14, 23] but this example will serve as a brief introduction to the approach. This example assumes ORR in acidic media following the associative reaction with sub-stepsgiven in Equations [4a-e]:

$$
\begin{aligned}
& { }_{+}^{*} \mathrm{O}_{2(\mathrm{~g})}+4 \mathrm{H}^{+}+4 e^{-} \rightarrow{ }^{*} \mathrm{O}_{2}+4 \mathrm{H}^{+}+4 e^{-} \\
& { }^{*} \mathrm{O}_{2}+4 \mathrm{H}^{+}+4 e^{-} \rightarrow{ }^{*} \mathrm{OOH}+3 \mathrm{H}^{+}+3 e^{-} \\
& { }^{*} \mathrm{OOH}+3 \mathrm{H}^{+}+3 e^{-} \rightarrow{ }^{*} \mathrm{O}+2 \mathrm{H}^{+}+2 e^{-}+\mathrm{H}_{2} \mathrm{O} \\
& { }^{*} \mathrm{O}+2 \mathrm{H}^{+}+2 e^{-}+\mathrm{H}_{2} \mathrm{O} \rightarrow{ }^{*} \mathrm{OH}+\mathrm{H}^{+}+e^{-}+\mathrm{H}_{2} \mathrm{O} \\
& { }^{*} \mathrm{OH}+\mathrm{H}^{+}+e^{-}+\mathrm{H}_{2} \mathrm{O} \rightarrow{ }^{*}+2 \mathrm{H}_{2} \mathrm{O}
\end{aligned}
$$

In these equations, ${ }^{*}$ indicates the active site and ${ }^{*} X$ represents chemical moiety $X$ bound to the active site. This reaction is composed of one initial chemical reaction $\left(\mathrm{O}_{2}\right.$ binding to the active site, [4a]) and four subsequent protonation reactions [4b-e], leading to generation of $2 \mathrm{H}_{2} \mathrm{O}$ molecules from one $\mathrm{O}_{2}$ molecule, 4 protons, and 4 electrons. To apply the $\mathrm{CHE}$, several assumptions are made. The final state containing the active site $(E *)$ and $2 \mathrm{H}_{2} \mathrm{O}$ molecules $\left(2 E_{H 2 O}\right)$ is taken to be $0 \mathrm{eV}$ to set the energy scale. The energy of this state is calculated by adding the calculated energies of the active site, ${ }^{*}$, with the energies of $2 \mathrm{H}_{2} \mathrm{O}$ molecules. The initial state is assumed to be $4.92 \mathrm{eV}$ higher than the final state at standard conditions, based on the known thermodynamics of the ORR and consistent with a standard redox potential of $1.23 \mathrm{~V} v s$. SHE for the 4 electron reaction [7].To set the electric potential, an assumption is made that at a potential of $0 \mathrm{~V}(U=0 \mathrm{~V})$, that two protons and two electrons are degenerate in energy to an $\mathrm{H}_{2}$ molecule at a $p H$ of zero. This assumption is analogous to the experimental use of a hydrogen-based reference electrode, leading to the term computational hydrogen electrode. The contribution to system energy of the protons and electrons at each sub-step as a function of electric potential is thus accessible via calculation of the $\mathrm{H}_{2}$ molecule. If a non-zero potential is considered, then the system energy is decreased by the considered potential, multiplied by the number of electrons at that state. Hence, higher 
potentials stabilize states with electrons and at a potential of $1.23 \mathrm{~V}$ (and standard conditions), the initial and final states are in thermodynamic equilibrium.In an ideal pathway, all considered states are in equilibrium, leading to no thermodynamic barriers up to $1.23 \mathrm{~V} v s$. CHE.Many studies have focused on acidic environments where fuel cell membrane technology is most mature. Reaction pathways and, subsequently, relative ORR activities, are likely to be different in basic environments. This difference is exemplified by the relatively high ORR activity of Pt in acidic environments but relatively moderate activity in basic environments.

For the above assumed reaction pathway, calculation of the $\mathrm{H}_{2}$ molecule state, $\mathrm{H}_{2} \mathrm{O}$ molecule state, the active-site structure $*$, the *0O bound structure, the * $\mathrm{OOH}$ bound structure, the ${ }^{*} \mathrm{O}$ bound structure, and the ${ }^{*} \mathrm{OH}$ bound structure are required. The initial and final states are fixed (for standard conditions), as discussed. For the other sub-step states, a system energy relative to the final energy is calculated. For instance, for the second sub-step that contains the *00 structure, 4 protons, and 4 electrons, DFT is used to calculate the energy of the *00 bound structure $\left(E^{*} O 0\right)$ and the energy of $2 \mathrm{H}_{2}$ molecules is added to take into account the 4 protons and electrons. The calculated system energy for the final state is then subtracted off giving the relative reaction energy for the second sub-step. Similarly, the ${ }^{*} \mathrm{OOH},{ }^{*} \mathrm{O}$, and ${ }^{*} \mathrm{OH}$ bound structures, along with the appropriate contribution to system energy from the $\mathrm{H}_{2}$ and $\mathrm{H}_{2} \mathrm{O}$, can be used to obtain the relative reaction energy for reaction coordinates 3,4 , and 5 . These relative reaction energies represent the reaction pathway at $U=0 \mathrm{~V}$.

Since the number of electrons at each state is known for the assumed pathway, a shift in potential can be applied after the calculations. There is an implicit assumption in thismethod that ORR intermediate binding energy is not directly affected by this shift in potential and that the dominant effect is due to the electrons present at each of the sub-steps.In this formalism, there is some applied potential, at which two of the sub-steps will be degenerate and, due to the decreasing number of electrons at increasing reaction coordinate (sub-steps farther along the reaction pathway), above this potential, there will always be at least one (and possibly multiple) thermodynamic barrier between the sub-steps. This value is called the limiting potential, $U_{l, \text { as }}$ described above and can serve as a DFT-accessible descriptor of ORR activity. Structures with higher calculated $U_{l}$ values do not have thermodynamic barriers in their reaction pathways until subjected to higher

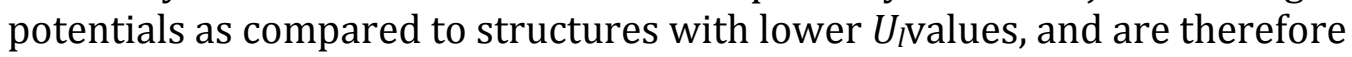
predicted to have higher activities. Correspondingly, at a fixed potential above the $U_{l}$ value of two sites, the thermodynamic barrier in the ORR reaction pathway is smaller for the site with the higher $U_{l}$ value and larger for the site with the lower $U_{l}$ value. The reaction that becomes degenerate at the limiting potential is called the potential-determining step and is important for understanding what is limiting the ORR. General trends based on limiting potentials and their potential-determining steps can be drawn. Further interrelations between sub-steps, known as scaling laws, have been demonstrated but are beyond the scope of the present work.

Clearly, this approach contains a multitude of approximations, as does any model attempting to capture complex behavior, but provides a computationally accessible method for linking active-site structures to ORR activity for comparison 
purposes. Other reaction pathways can be considered through variations in calculated sub-states. Deviations from ideal and standard conditions can also be introduced. Sensitivity to model choices/assumptions can be explored and a more uniform methodology, enabling comparison between calculations of different research groups, are all possible due to this usefulcomputational approach.

\subsection{Metal-Bearing Active Sites}

Active-sitestructures considered in literature can be broken down into sites that contain transition metals (metal-bearing) and those that do not (metal-free). It is well documented that materials synthesized with metal precursors lead to significantly more active ORR catalysts[35] but it is possible that either the metal itself is a central part of the active site or acts to catalyze/template formation of specific ORR active $\mathrm{C}-\mathrm{N}$ structures. This distinction is made more complicated due to trace amounts of transition metals present in many "metal-free" materials, as discussed by Wang et al.[36]. Another complication arises when a two-site ORR reaction occurs, in which one site generates $\mathrm{H}_{2} \mathrm{O}_{2}$ that is further reduced to two water molecules on a secondary site[37].In contrast to " $4 \mathrm{e}^{-}$pathways", this " $2+2 \mathrm{e}^{-}$ pathway" is likely to generate significantly more $\mathrm{H}_{2} \mathrm{O}_{2}$, which may degrade fuel cell polymer membranes. DFT can be useful for determining which pathway is thermodynamically favored and thus gives clues to the selectivity of a given activesite structure toward preferable 4 e- pathways[14].

Even if such complications are avoided and a single active site following a given $4 \mathrm{e}$ - pathway with a transition metal(TM) as the ORR intermediate adsorption site is assumed, a variety of structural questions still remain. How does $\mathrm{N}$ coordination of TM affect activity? How does local carbon-support structure affect activity? Which transition metals make the best ORR catalysts? In general, what is theoptimum TM-N-C active-sitestructure and how can this knowledge be used to synthesize better non-PGM catalyst materials? While many of these questions remain unanswered, we will discuss here some of the progress that has been reported using DFT to better understand how TM-N-C structure couples to ORR activity.

Calle-Vallejoet al.[13] report calculations of ORR via a $4 \mathrm{e}^{-}$associative pathway with no initial chemical adsorption of $\mathrm{O}_{2}$ on two $\mathrm{M}-\mathrm{N}_{4}$ bulk graphene structures with varied metal (M) species (including non-transition-metals, $\mathrm{M}=\mathrm{Cr}$, $\mathrm{Mn}, \mathrm{Fe}, \mathrm{Ru}, \mathrm{Co}, \mathrm{Rh}, \mathrm{Ir}, \mathrm{Ni}, \mathrm{Pd}, \mathrm{Pt}, \mathrm{Cu}, \mathrm{Ag}$, and $\mathrm{Au}$ ). The first structure (Cell A) contained 5 member rings coordinating $\mathrm{M}$ and the second structure (Cell $\mathrm{B}$ ) places $\mathrm{M}$ at the center of an N-coordinated C divacancy (analogous to the $\mathrm{FeN}_{4}$ structure discussed previously in the Stability section). These structures are shown in Figure 5. Mn, Fe, and Co M-species were calculated to be the most ORR-active $\mathrm{M}-\mathrm{N}_{4}$ sites. Cell B was reported to be more active than Cell A for Co and Cell A more active for Mn and Fe. These findings highlight the sensitivity ofORR activity to transition metal species, as well as to local carbon structure, as represented by the two different cell structures.

Kattelet al.[25] demonstrated that other transition metal species may have alternate active structures, as compared to the more canonical graphitic (embedded) $\mathrm{M}-\mathrm{N}_{4}$ sites. They considered the activities of a Ni-N $\mathrm{N}_{4}$ graphene bulk 
system, as well as a $\mathrm{Ni}-\mathrm{N}_{2}$ nanoribbon armchair edge structure, with the Ni adsorbed at an edge, and reported that the edge structure is a more likely candidate for the ORR active site in acidic media, based on calculated activity. The same authors also considered how N-coordination of graphene-embedded Co affects ORR activity and selectivity[26]. Based on stability constraints from DFT calculated formation energies, a 4-fold $\mathrm{N}$-coordination is thermodynamically likely but this structure is predicted to preferentially generate $\mathrm{H}_{2} \mathrm{O}_{2}$, requiring a second active site for the $2+2$ e-ORR reaction. These studies again highlight the importance of atomic-scale structure, including $\mathrm{N}$-coordination, on ORR activity and add the consideration that active sites at or near edges may behave differently than those contained in the bulk of the graphene structure.

A correlation between ORR activity and porosity of materials has been identified experimentally[3,4], prompting two groups to consider the formation and activity of TM-N 4 structures in and near models of such pores. Kattel and Wang[27]reported that, similarly to the stabilization of defects near edges discussed above, TM-N $\mathrm{N}_{4}$ defects are structurally stabilized in pore-like structurescompared to bulk graphene. Szakacs et al.[16] also suggest that, unlike graphene bulk structures that predominantly contain flat $\mathrm{Fe}-\mathrm{N}_{4}$ defects, pore-supported defects may distort out of plane, altering the local structure. Such defects, when incorporated into bulk graphene, are found to be flat/planar to the graphene host. Both groups consider $\mathrm{O}_{2}$ binding as a function of structure and determine that the final protonation step from ${ }^{*} \mathrm{OH}$ to $\mathrm{H}_{2} \mathrm{O}$ is likely the potential-determining step in such structures. Szakacs et al. also consider the idea of a ligand modification of the Fe-N 4 pore site and its effect on calculated ORR, an idea proposed by Zhu et al.[38]for shifting $\mathrm{O}_{2}$ binding energies in non-PGM active sites. They determine that this ligand can alter the reaction pathway.This suggests that understanding of ligand interaction/modification of active sites may be a valuable tool in optimizing stability, ORR activity, and selectivity.

Following previous studies considering structural stability, Holby and Taylor calculated ORR activity on an $\mathrm{Fe}_{2} \mathrm{~N}_{5}$ zig-zag edge structure (Figure 6), finding that a strongly bound ${ }^{*} \mathrm{OH}$ in the $\mathrm{ORR}$ reaction pathway is likely to be persistent atrelevant potentials[24]. Similar to the works of Zhu et al. and Szakacs et al., this *OH was treated as a modifying ligand, albeit one that is calculated to form spontaneously during ORR. The spontaneous * $\mathrm{OH}$ ligand modification dramatically improved the ORR activity on the $\mathrm{Fe}_{2} \mathrm{~N}_{5}$ site, which, due to its 3-dimensional nature, was not blocked by the bound ${ }^{*} \mathrm{OH}$ as would occur on 2-dimensional metal surfaces. Applying this ligand effect to a mixed-metal FeCoN 5 zig-zag edge structure produced an active site with a predicted $U_{l}$ value of $0.80 \mathrm{~V}$, the highest calculated ORR activity for non-PGM structures that includes the chemical adsorption of $\mathrm{O}_{2}$. This effect has also been calculated to apply to a variety of TM- $\mathrm{N}_{4}$ edge structures and is likely a general effect for non-PGM active sites, in which the potential-determining step is the final protonation.

Another active-site structure, suggested based on thermodynamic stability, is the $\mathrm{Fe}_{2} \mathrm{~N}_{6}$ structure (Figure 7)[19].Unlike many other TM-bearing sites, this structure's calculated potential-determining step is the first protonation reaction due to the strong $\mathrm{O}_{2}$ binding. Owing to the more weakly bound * $\mathrm{OH}$ moiety, 
spontaneous $* \mathrm{OH}$ ligand modification is not predicted to occur on such structures at potentials below calculated limiting potentials. When hosted at a zig-zag edge, the limiting potential is calculated to be $0.37 \mathrm{~V}$. Compared to the $\mathrm{Fe}_{2} \mathrm{~N}_{5}$ structure, the $\mathrm{Fe}_{2} \mathrm{~N}_{6}$ structure is less strongly stabilized by proximity to an edge, which is attributed to decreased strain for the 4 -fold $\mathrm{N}$ coordinated structures that make up this site. The limitingpotential for the graphene-bulk-hosted structure is slightly improved with a calculated value of $0.42 \mathrm{~V}$. If, for the sake of argument, the initial $\mathrm{O}_{2}$ adsorption and first protonation step are combined into a single electrochemical reaction step, the calculated limiting potentials would be drastically increased to $0.91 \mathrm{~V}$ and $0.98 \mathrm{~V}$ for the zig-zag and bulk structures, respectively. This assumption is often made in literature (as will be discussed below) and is still a matter of debate. Such a dramatic difference in calculated activity in these two cases could be utilized to experimentally determine if an initial chemical step does occur (assuming it would be possible to synthesize such structures homogeneously).

It is clear that a diversity of active-site structures involving TMs may be formed during synthesis and, based on DFT calculations, these structures are likely to have widely varying ORR activity.N-coordination, planarity, position in relation to edge/pores, and modifying ligands have all been identified as potentially important contributors for linking ORR activity to metal-bearing active-sitestructures.

\subsection{Metal-Free Active Sites}

Even if transition metal precursors are required for synthesis of highly active nonPGM materials, that does not directly indicate that these metal atoms are at the center of active sites, as discussed in the previous section. While such sites can have quite high calculated ORR activity, that does not preclude other active sites that do not include TMs.TM atoms have been reported to catalyze formation of $\mathrm{C}$ vacancies[39], which may aid in doping of $\mathrm{N}$ or form more complex defect reconstructed structures, which in turn may possess high catalytic activity[29]. Formation of persistent TM-rich nanoparticles (possibly carbides, oxides, sulfides, nitrides, or metal), surrounded by protective carbon layers, have been observed in synthesized materials and some authors have suggested that this may template ORR active sites on the $\mathrm{C}$ surface[40-42] or alter ORR activity of $\mathrm{N}$-doped graphene[43]. Other groups, however, have synthesized materials without transition metal nanoparticle formation and without loss of high activity[44], suggesting this effect is not necessarily responsible for high ORR activity.Additionally, at least one DFT study [43] considering $\mathrm{N}$-doped $\mathrm{C}$ layers over a metal substrate determined a limiting potential similar to that reported for metal-free N-doped graphene [22], both significantly below the high limiting potentials calculated for metal-bearing sites discussed in section 3.1.Given that TM nanoparticles compete for TM precursor atoms during synthesis and do not lead to improved activity, future work should focus on synthesis routes that avoid formation of such nanoparticles.

Without assuming what role the transition metal atoms play in formation of active sites, a variety of truly metal-free structures have been suggested and their activity explored using DFT. Generally speaking, metal-free sites tend to bind ORR intermediates lessstrongly than metal-bearing sites, in many casesleading to the 
first protonation step being potential determining. In fact, for many $\mathrm{C}-\mathrm{N}$ structures, $\mathrm{O}_{2}$ does not exothermically bind but, instead, has been found to occupy a locally meta-stable position above the active site[29,45].Due to this effect, a reaction pathway similar to that in Equations [4a-e],but with the first two sub-reactions combined into a single binding/electron transfer step,is often assumed, leaving 4 electrochemical sub-reactions in the $4 \mathrm{e}^{-}$pathway (and no initial chemical step). In this new pathway, Equations [4a] and [4b] are replaced by Equation 5. If this assumption were not made, there would either be a thermodynamic barrier in the reaction pathway for all potentials due to the $\mathrm{O}_{2}$ meta-stability or, the reported limiting potentials would be incorrect since the first electrochemical step would be between the bound ${ }^{*} \mathrm{OO}$ state and the ${ }^{*} \mathrm{OOH}$ state, not the $\mathrm{O}_{2(\mathrm{~g})}$ state and the ${ }^{*} \mathrm{OOH}$ state. Since the first protonation step is often the potential determining step for metal-free sites, the calculation of the limiting potential is particularly sensitive to this choice. The validity of this assumption remains one of the outstanding considerations in the field of ORR active-site modeling. Because of this, particular attention is paid to consideration of $\mathrm{O}_{2}$-active site interactions when studying metal-free active-site structures. With the caveat of this assumption made, we present examples of metal-free active-site structures and DFT findings on how structure couples to ORR activity and attempt to provide details of the $\mathrm{O}_{2}$-active site interaction, where available. Generally, based on comparisons of $\mathrm{OOH}$ binding energies, the $\mathrm{C}$ adjacent to doped- $\mathrm{N}$ is found to serve as the intermediate binding site similar to the metal atom in the metal-bearing structures.

${ }^{*}+\mathrm{O}_{2(g)}+4 \mathrm{H}^{+}+4 e^{-} \rightarrow{ }^{*} \mathrm{OOH}+3 \mathrm{H}^{+}+3 e^{-}$

Okamotoused first-principles molecular dynamics based on DFT to study ORR on N-doped graphene structures[46]. In this work, it was determined that increased $\mathrm{N}$-doping leads to increased $\mathrm{O}_{2}$ binding and that doping values above 9 at.\% $\mathrm{N}$ ( 3 and $4 \mathrm{~N}$ atoms in their $32 \mathrm{C}$ unit cell) were required for $\mathrm{O}_{2}$ to adsorb exothermically. Furthermore, high $\mathrm{N}$-content structures were comprised of second nearest-neighbor $\mathrm{N}$ clusters, which were shown by Kwak et al.[20] to be not representative of stable $\mathrm{C}-\mathrm{N}$ phases, up to 11.1 at.\% N.Thus, such structures would only be kinetically stable. Using a different electrochemical model for ORR activity via an associative pathway, Okamoto predicted that these highly $\mathrm{N}$-doped materials are likely to have minimum reversible potentials (analogous to $U_{l}$ ) of $0.34 \mathrm{~V}$ for bulk and $0.39 \mathrm{~V}$ for edges with protonation of adsorbed ${ }^{*} O$ being the limiting step in the considered water environment. These findings highlight one of the central issues faced by metal-free active sites: structures that bind $\mathrm{O}_{2}$ exothermically are rare and do not appear to have very ideal reaction pathways.

Kim et al.also considered ORR on N-doped graphene structures and proposed a novel ring-opening pathway,in which the adsorbed ${ }^{*} \mathrm{OOH}$ cleaves a $\mathrm{C}-\mathrm{N}$ bond near zig-zag edges[28]. This pathway shifts the minimum reversible potential step, creating a more favorable ORR pathway. The considered N-doping site, however, is not consistent with calculated thermodynamically favorable $\mathrm{N}$ doping positionsof such edges and the stable structures (with $\mathrm{N}$ occupying the outermost $\mathrm{C}$ of the zig- 
zag edge) were not tested. The proposedring-opening reaction pathway was reported by Kwak et al.[20] for highly $\mathrm{N}$-doped ( $\sim 27.8$ at.\% $\mathrm{N})$ edge structures that are consistent with $\mathrm{N}$ at the outer zig-zag edge but the authorsconsidered the structure susceptible to degradation and did not report limiting potential values for this structure.

In addition to this high $\mathrm{N}$-doping zig-zag structure, Kwak et al. considered lower N-doping of graphene sheets and zig-zag edged nanoribbons. These structures all fall on the calculated thermodynamic convex hull and thus represent the thermodynamically predicted structures for N-doped bulk and zig-zag edge nanoribbons. The authors reported that bulk structures are more ORR-active than edge structures due to stronger * $O$ binding at edges. For bulk graphene structures, they reported a very strong dependence of limiting potential on $\mathrm{N}$-doping and that in general, the lower the $\mathrm{N}$-doping, the more active the material (higher $U_{l}$ ). A recent review of experimental data of synthesized (metal-bearing) materials with the highest ORR mass activity showed no significant trend relating $\mathrm{N}$-contentto activity but significantly lower $\mathrm{N}$-doping levels (up to $\sim 4$ at. $\% \mathrm{~N}$ ) were reported[4]. This indicates that perhaps this trend only occurs for more highly $\mathrm{N}$-doped materials and also suggests that further increasing of $\mathrm{N}$-doping concentrations is not ideal for improving ORR activity.

Changes in reaction pathways with the addition of solvation effectsare shown by Kwak et al.[20] to have a large impact on reported limiting potentials, as the solvation stabilizes ${ }^{*} \mathrm{OOH}$ binding (or ${ }^{*} \mathrm{O}+\mathrm{OH}$ binding) which is integral to their potential determining step. Disregarding a chemical *00 adsorption step, they found a maximum limiting potential of $0.79 \mathrm{~V}$ for solvated 5.5 at.\% $\mathrm{N}$-doped graphene structures. The same structure without solvation effects was calculated to have a limiting potential of $0.55 \mathrm{~V}$. This value is very close to the value reported by Studt, who reported a limiting potential of $0.51 \mathrm{~V}$ on a 6.3 at.\% $\mathrm{N}$-doped graphene sheet without solvation effects[23]. The stated potential determining step, however, differs between these two studies with Kwak et al. reporting the first protonation step to be potential determining and Studt reporting the protonation of ${ }^{*} 0$ to be potential determining (third protonation step). There are several reasons that may explain these variations (underlying choices of DFT, different N-doped structures, etc.) but the possibility of solvation environment affecting reported limiting potentials is an important consideration.

Lyalin et al.considered the concurrent contributions of solvation, zero-point energy, and vibrational entropy on binding energies of ORR intermediates on B-N structures and found that for most intermediates (with the exception of a $* \mathrm{O}+{ }^{*} \mathrm{OH}$ state), these effects largely cancelled each other out[33]. This finding is consistent with Anderson, who reported that using uncorrected DFT calculated gas-phase binding from internalenergy values for determining free energies of intermediate states (no zero-point energy, no entropic effects, no solvation effects, and no charge/double layer effects) produced at most a $0.2 \mathrm{~V}$ error in calculation of reversible potentials[12].Further work, particularly closely linked theoretical and experimental studies on well-defined systems, will greatly aid in understanding what role both $\mathrm{N}$-content and solvation environment play in determining ORR 
activity and what modifications DFT-based models may require to capture these effects.

Formation of $\mathrm{C}$ vacancies and associated defects may be one way in which transition metal atoms aid in formation of active sites without being a part of the active site itself. Chai et al. considered ORR activity for a host of $\mathrm{N}$-doped graphene, graphene edge and nanotube structures with and without relaxed C-vacancy structures, including Stone-Wales (SW) defects. They found that defects could significantly alter $\mathrm{O}_{2}$ interaction with the surface but that for all considered sites, $\mathrm{O}_{2}$ did not bind exothermically but rather occupied a meta-stable site above the surface.Thus, the direct protonation of $\mathrm{O}_{2(\mathrm{~g})}$ to form ${ }^{*} \mathrm{OOH}$ was considered without

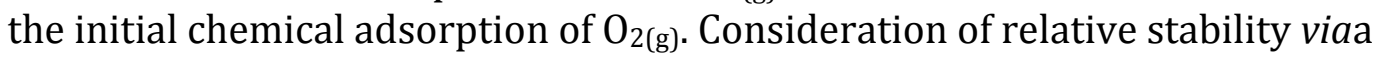
calculated convex hull as a function of N-doping of a SW defect in graphene indicated that a doping level of $\sim 3.3$ at $\% \mathrm{~N}$ (with $\mathrm{N}$ in well-defined positions in relation to the defect) was thermodynamically most favorable, with lower and higher doping levels being less thermodynamically stable. For bulk graphene, this site had a calculated limiting potential of $0.55 \mathrm{~V}$, similar to $\mathrm{N}$-doped graphene in other studies. Graphene structures with multiple $\mathrm{N}$ in the neighboring carbon ring were found to have a lower limiting potential of $\sim 0.32 \mathrm{~V}$, suggesting a deleterious effect of additional local $\mathrm{N}$ on activity, as also suggested by Kwak et al.[20]in their study of N-doped graphene.

Chai et al. also considered the effect of curvature on limiting potential[29]. They discovered through the simulation of nanotubes of different radii that curvature (and likely induced strain that it causes) could be used to tune binding energy and thus alter the calculated limiting potential value since the potential determining step was sensitive to choice of radius. They found that by embedding their most stable SW defect with $2 \mathrm{~N}$ in a $(10,0)$ nanotube that the limiting potential, calculated without intermediate $\mathrm{O}_{2}$ binding, could be increased to $0.80 \mathrm{~V}$. Their data demonstrates a "volcano plot" for activity as a function of nanotube radius, exemplifying the Sabatier principle in which nanotube radius controls relative binding and shifts from overbinding to underbinding with some peak, activity maximizing value. The strain-engineering of catalysts has been previously applied to other systems[47] and holds great promise for increasing the ORR activity of nonPGM catalysts.

Similar to metal-bearingnon-PGM sites, metal-free site ORR activities are clearly dependent upon not only $\mathrm{N}$-doping concentrations but also local C-N configurations. While the issue of the binding of $\mathrm{O}_{2}$ to such sites remains, even for optimized N-doped structures, C-defect and strain-engineering are demonstrated to be useful tools for tuning ORR activity and, along with theapproaches discussed in the metal-bearing catalyst section, will provide useful pathways for improving the ORR activity of synthesized non-PGM ORR materials.

\section{Linking Experimental Signatures and Structures}

The previous sections discuss how DFT is utilized in a bottom-up approach to determine the atomic-scale nature of sites that are both stable relative to other active-site candidates and highly ORR active. With enhanced understanding of such 
sites, it is possible for DFT modeling to help guide synthesis efforts in order to maximize the number of such sites in real, synthesized materials. However, this connection between desired active site and realized active site from synthesis can be difficult to make. As a variety of increasingly active and durable catalysts are being synthesized, it is also possible to utilize DFT in a top-down approach inorder to better understand the relationship between the experimental signatures produced by these ORR-active synthesized materials and the atomic-scale nature of the active sites that they contain. We briefly discuss studies that utilize this approach in order to demonstrate usefulness of such efforts and how DFT can aid in interpretation of experimental data. It should be pointed out, however, that there is a fundamental hurdle faced universally in the field of catalysis that is particularly pertinent to such studies. High catalytic activity may be due to numerous moderately active sites (which may dominate experimental signatures) or the activity may be dominated by relatively rare sites with exceptionally high catalytic activity (which may not appear dominant in experimental signatures). Thus, it is important to couple trends from a given experimental signature with observed catalytic activity when possible.

The above DFT studies suggest that for metal-bearing active sites, ligand modification may be an important component of explaining ORR activity. Such ligand effects should have experimental signatures. For instance, Szakacs et al. suggest that Mößbauer spectroscopy spin-state signatures of highly active synthesized metal-bearing catalyst materials can be explained by a combination of agiven active sites with and without a $5^{\text {th }}$ axial ligandwhich DFT calculates to have nearly degenerate formation energies[16]. Jia et al.[48] suggest a switching behavior that is accompanied by a decrease in Fe-N bond distance during the ORR reaction as measured by in-situ EXAFS for a highly ORR active PANI-Fe-C catalyst. A less active phthalocyanine-based catalyst displayed a lengthening of the Fe- $\mathrm{N}$ bond distance under ORR conditions. DFT calculations of $\mathrm{FeN}_{4}$ with and without $\mathrm{NH}_{2}$ ligand predicted both an increase in calculated ORR activity with ligand $v s$. ligand-free structuresand adecrease in Fe-N bond length during ORR due to adsorption of intermediates opposite the ligand, pulling the central Fe atom back toward the graphene host plane. Zitolo et al. demonstrated that XANES data is best fit by $\mathrm{FeN}_{4}$ structures, in which multiple $\mathrm{O}_{2}$ molecules are bound to the Fe site, suggesting the possibility of ligand occupation. Subsequent DFT calculations indicate that $\mathrm{O}_{2}$ binding energies are sensitive to an initial ligand (in their case, $\mathrm{O}_{2}$ ) [44]. This suggests that the ORR pathway may be affected by the presence of ligands that are themselves ORR intermediates, as suggested earlier by Holby and Taylor based on DFT calculations [24].While the exact chemical nature of active-site ligand modification is still under consideration, a growing amount of both DFT and experimental literature suggest that such an effect appears to be an important aspect of highly ORR-active materials and provides a potential path forward for tuning active sites toward higher ORR activity.

$\mathrm{X}$-ray photoelectron spectroscopy (XPS)represents a valuable tool for identifying the atomic-scale nature of non-PGM active-site structures. However, deconvolution of experimentally derived XPS data depends on fitting to models of binding energy spectra. In some cases, these models have been obtained from 
known structural sources but in cases where no such model exits, DFT can be utilized for generation of such a model. Artyushkova et al. demonstrated the validity of such an approach to TM- $\mathrm{N}_{\mathrm{x}}$ structures, through calculation of the $\mathrm{N} 1 \mathrm{~s}$ core electron shifts in comparison to known model structures[49].Kabiret al.applied this approach to several $\mathrm{Fe} / \mathrm{Co}-\mathrm{N}_{3}$ structures, showing that their calculated $\mathrm{N} 1 \mathrm{~s}$ core level shift is consistent with XPS data from active ORR catalyst materials, thus suggesting the feasibility of such structures as active sites[21].

The above efforts demonstrate how DFT studies can aid in the interpretation of experimental data and relate experimental signatures to atomic-scale structures. Other surface-sensitive approaches are being actively pursued for identification of synthesized ORR catalysts and DFT will almost certainly remain a useful tool for interpreting the signatures generated by these highly heterogeneous materials in experimental studies.

\section{Future Directions}

The application of DFT modeling is greatly aiding in the effort to understand the atomic-scale structures in pyrolyzed TM-N-C and $\mathrm{N}-\mathrm{Cmaterials}$ that give rise to very high ORR activities. Active sites with calculated activities rivaling that of Pt have been demonstrated and future studies will aid in further increasing activity, durability, and 4e- pathway selectivity. Models of catalyst corrosion and poisoning mechanisms are required to fully understand how active sites are lost during fuel cell operation. With the increased knowledge of the atomic-scale structures likely being responsible for ORR activity, such models are now within reach. Further understanding of the link between active sites and modifying ligands may provide a path forward for carefully tuning ORR activity and selectivity. Intentional integration of optimized ligand moieties into catalytic materials may enable catalysts even more active than Pt. Improved modeling techniques,combining the diverse variety of physical and chemical phenomena experienced under fuel cell operating conditions may open further possibilities for improved eletrocatalysts and aid in settling the now 60 year old conundrum of what are the structures responsible for ORR in TM-N-C materials and how can these structures be further optimized to enable cost-effective fuel cell technology.

\section{Vitae}

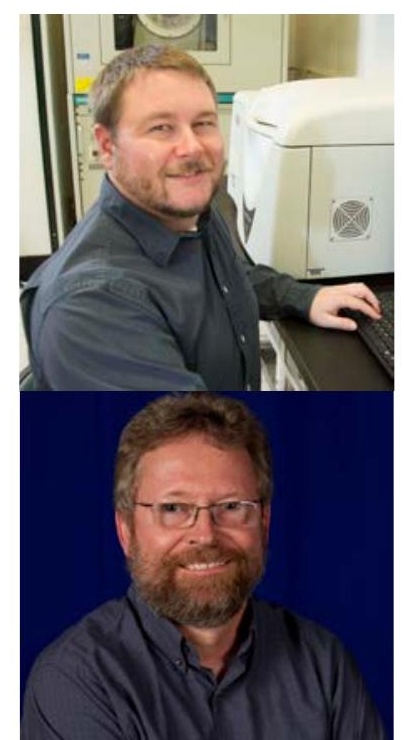

Edward (Ted) F. Holby is a research scientist in the Sigma Division of Los Alamos National Laboratory. He is a graduate of the University of Wisconsin - Madison (Ph.D. in Materials Science, 2011) and Carleton College (M.A. in Physics and Astronomy, 2004). His research is focused on the theory and modeling of catalysis and corrosion of materials for energy storage and conversion devices.

Piotr Zelenay received his Ph.D. and D.Sc. ("habilitation") degrees in chemistry from the University of Warsaw, 
Warsaw, Poland. He was a faculty member at the University until 1997, when he accepted a permanent research position with Los Alamos National Laboratory (LANL). As a project and team leader, he has been associated with Materials Physics and Applications Division at Los Alamos for the past 19 years. Recently, Dr. Zelenay was appointed a Laboratory Fellowby LANL. Dr. Zelenay's research focuses primarily on fundamental and applied aspects of polymer electrolyte fuel cell science and technology, electrocatalysis, and electrode kinetics.

\section{Acknowledgements}

Financial support from the Office of Energy Efficiency and Renewable Energy of the U.S. Department of Energy through Fuel Cell Technologies Office and from Los Alamos National Laboratory through Laboratory Directed Research and Development is gratefully acknowledged. E.H. would like to thank the LANL Institutional Computing Program for computational support. 


\section{Figures}

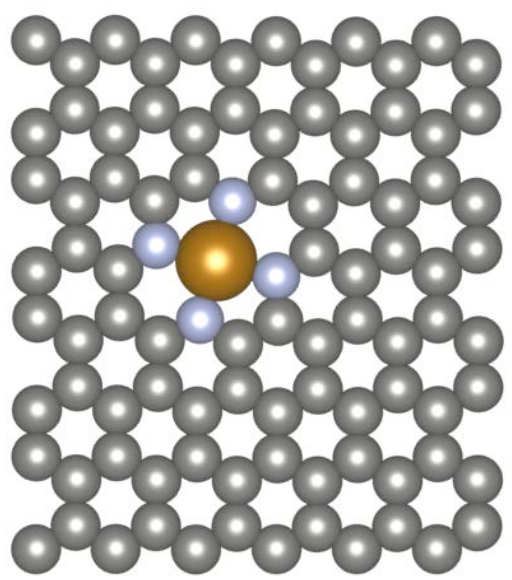

Figure 1: $\mathrm{FeN}_{4}$ structure with $\mathrm{Fe}$ in $\mathrm{C}$ bivacancy, $\mathrm{N}$ substituting for 4 neighboring $\mathrm{C}$ atoms and a periodic graphene sheet structure as viewed from above. Gray spheres represent $\mathrm{C}$, blue spheres represent $\mathrm{N}$, and the bronze sphere represents Fe. 


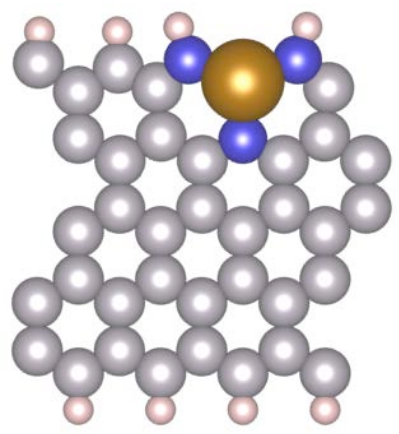

Figure 2. $\mathrm{FeN}_{3}$ structure at a zig-zag edge with Fe in $\mathrm{C}$ vacancy, $\mathrm{N}$ substituting for 3 neighboring $\mathrm{C}$ atoms and a periodic nanoribbon structure, as viewed from above. Gray spheres represent $\mathrm{C}$, blue spheres represent $\mathrm{N}$, and the bronze sphere represents Fe. 


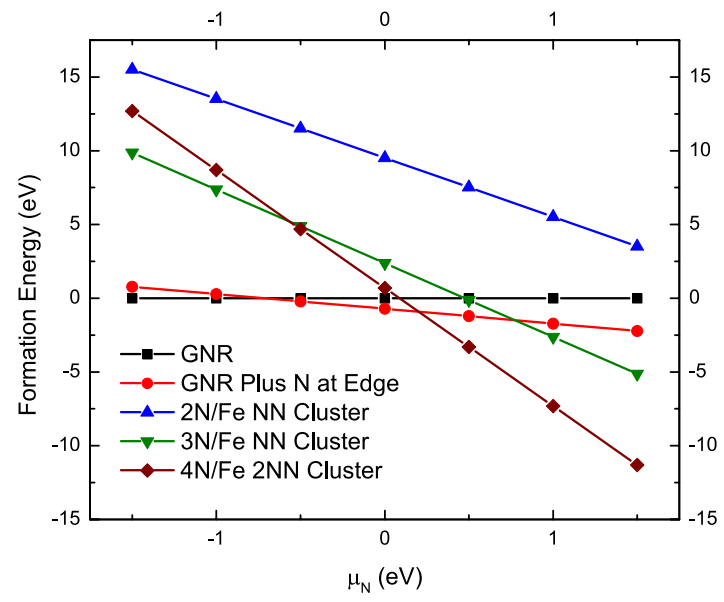

Figure 3: Stability plot of formation energy of different $\mathrm{N}$ and Fe/N zig-zag edge nanoribbon structures as a function of $\mathrm{N}$ chemical potential at a fixed Fe chemical potential (from reference [19]). GNR is the defect-free nanoribbon and other structures represent chosen $\mathrm{C}-\mathrm{N}$ and $\mathrm{C}-\mathrm{N}-\mathrm{Fe}$ structures. This plot emphasizes how changes in chemical potential affects which phase is calculated to be most stable (lowest on the plot at a given $\mathrm{N}$ chemical potential). 


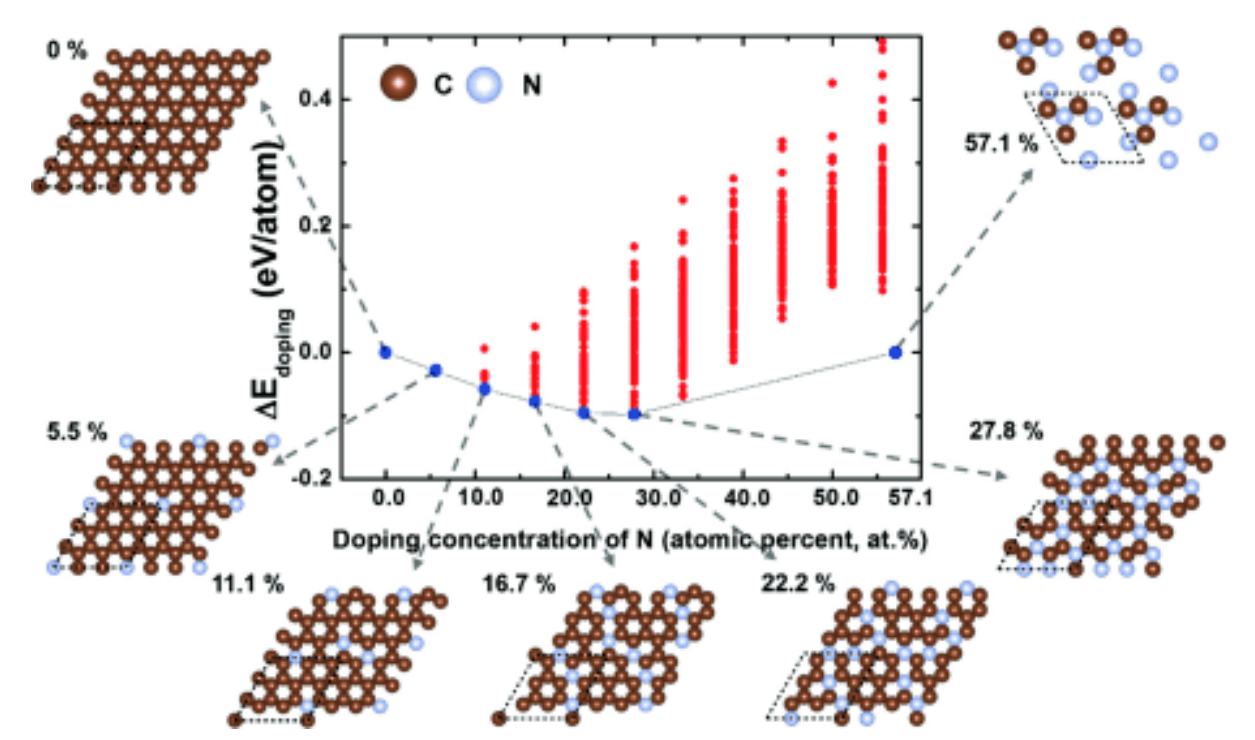

Figure 4:DFT-calculated convex hull of N-doped graphene (from reference [20]). Such studies provide thermodynamic insight regarding relative stability of atomic arrangements. Of particular interest from this study is the finding that $\mathrm{N}$-doped atoms do not prefer neighboring positions. 


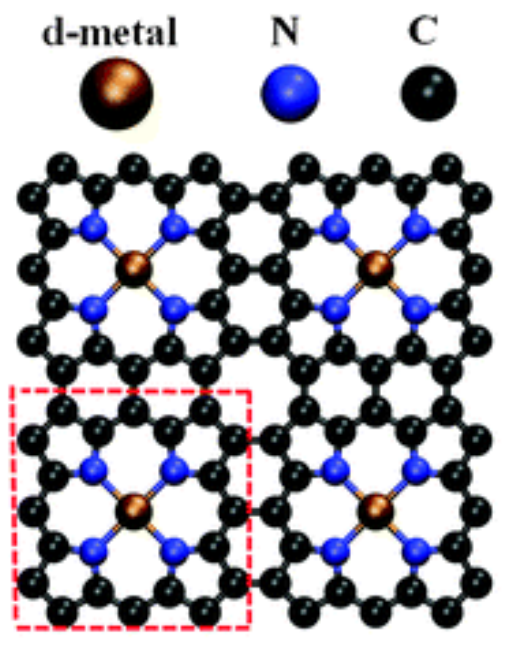

a) Cell $\mathrm{A}$

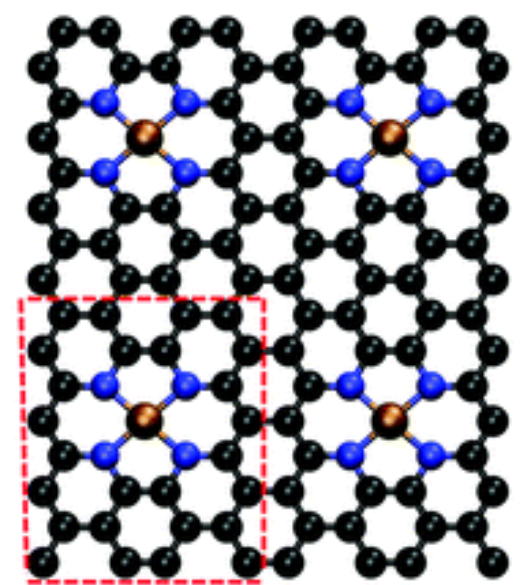

b) Cell B

Figure 5:Two different implementations of $\mathrm{M}-\mathrm{N}_{4}$ structures utilized in reference [13]. Changes in the local $\mathrm{C}$ environment were found to impact relative stability of structures as well as their adsorption characteristics (and thus activity). Changes in central metal atom also had a marked effect on predicted ORR activity. 


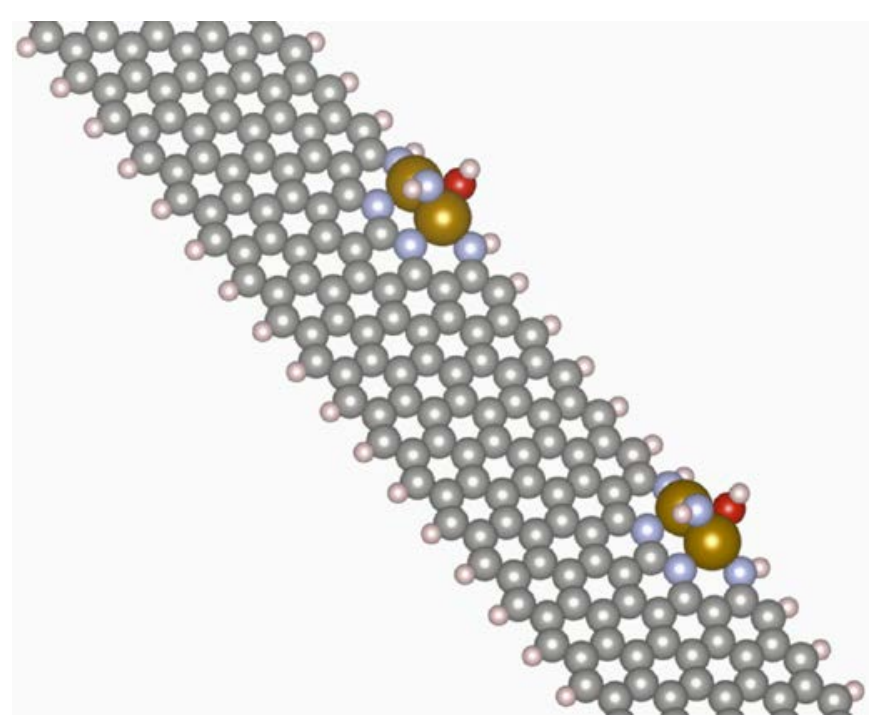

Figure 6: $\mathrm{Fe}_{2} \mathrm{~N}_{5}\left({ }^{*} \mathrm{OH}\right)$ structure utilized in reference [24]. This and other structures exhibit a spontaneous ${ }^{*} \mathrm{OH}$ ligand modification effect in which a persistent ${ }^{*} \mathrm{OH}$ ligand is likely to be formed at potentials below the calculated limiting potential. This modification modifies ORR intermediate adsorption energies and thus the activity of these sites. 


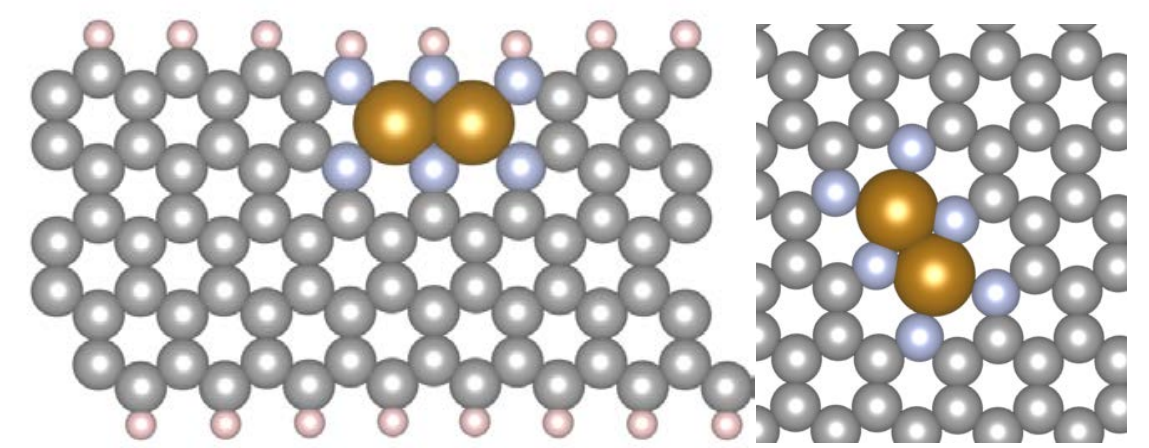

Figure 7: $\mathrm{Fe}_{2} \mathrm{~N}_{6}$ zig-zag and bulk structures. Such structures and their activities may be useful for determining if the chemical adsorption of $\mathrm{O}_{2}$ is the initial step in the ORR on non-PGM catalysts. 


\section{References}

[1] R. Jasinski,Nature, 201 (1964) 1212-1213.

[2] S. Gupta, D. Tryk, I. Bae, W. Aldred, E. Yeager, Journal of Applied Electrochemistry 19 (1989) 19-27.

[3] M. Lefevre, E. Proietti, F. Jaouen, J.-P. Dodelet, Science 324 (2009) 71-74.

[4] F. Jaouen, E. Proietti, M. Lefevre, R. Chenitz, J.-P. Dodelet, G. Wu, H.T. Chung, C.M. Johnston, P. Zelenay, Energy \& Environmental Science 4 (2011) 114-130.

[5] G. Wu, K.L. More, C.M. Johnston, P. Zelenay, Science 332 (2011) 443-447.

[6] Z. Chen, D. Higgins, A. Yu, L. Zhang, J. Zhang, Energy \& Environmental Science 4 (2011) 3167-3192.

[7] G. Wu, A. Santandreu, W. Kellogg, S. Gupta, O. Ogoke, H. Zhang, H.-L. Wang, L. Dai, , Nano Energy (present issue).

[8] J.K. Norskov, J. Rossmeisl, A. Logadottir, L. Lindqvist, J.R. Kitchin, T. Bligaard, H. Jonsson, Journal of Physical Chemistry B 108 (2004) 17886-17892.

[9] F. Abild-Pedersen, J. Greeley, F. Studt, J. Rossmeisl, T.R. Munter, P.G. Moses, E. Skulason, T. Bligaard, J.K. Norskov, Physical Review Letters 99 (2007) 016105. [10] J.K. Norskov, T. Bligaard, J. Rossmeisl, C.H. Christensen, Nature Chemistry 1 (2009) 37-46.

[11] A.B. Anderson, J. Uddin, R. Jinnouchi, The Journal of Physical Chemistry C 114 (2010) 14946-14952.

[12] A.B. Anderson, Physical Chemistry Chemical Physics 14 (2012) 1330-1338.

[13] F. Calle-Vallejo, J.I. Martinez, J. Rossmeisl, Physical Chemistry Chemical Physics 13 (2011) 15639-15643.

[14] I.C. Man, H.-Y. Su, F. Calle-Vallejo, H.A. Hansen, J.I. Martínez, N.G. Inoglu, J.

Kitchin, T.F. Jaramillo, J.K. Nørskov, J. Rossmeisl, ChemCatChem 3 (2011) 1159-1165. [15] S. Kattel, P. Atanassov, B. Kiefer, The Journal of Physical Chemistry C 116 (2012) 8161-8166.

[16] C.E. Szakacs, M. Lefevre, U.I. Kramm, J.-P. Dodelet, F. Vidal, Physical Chemistry Chemical Physics 16 (2014) 13654-13661.

[17] E.F. Holby, C.D. Taylor, Applied Physics Letters 101 (2012) 064102.

[18] E.F. Holby, G. Wu, P. Zelenay, C.D. Taylor, ECS Transactions 50 (2012) 18391845.

[19] E.F. Holby, G. Wu, P. Zelenay, C.D. Taylor, The Journal of Physical Chemistry C 118 (2014) 14388-14393.

[20] D. Kwak, A. Khetan, S. Noh, H. Pitsch, B. Han, ChemCatChem 6 (2014) 26622670.

[21] S. Kabir, K. Artyushkova, B. Kiefer, P. Atanassov, Physical Chemistry Chemical Physics 17 (2015) 17785-17789.

[22] S. Kattel, P. Atanassov, B. Kiefer, Physical Chemistry Chemical Physics 16 (2014) 13800-13806.

[23] F. Studt, Catalysis Letters 143 (2013) 58-60.

[24] E.F. Holby, C.D. Taylor, Scientific Repeports 5 (2015).

[25] S. Kattel, P. Atanassov, B. Kiefer, The Journal of Physical Chemistry C 116 (2012) 17378-17383. 
[26] S. Kattel, P. Atanassov, B. Kiefer, Physical Chemistry Chemical Physics 15 (2013) 148-153.

[27] S. Kattel, G. Wang, Journal of Materials Chemistry A 1 (2013) 10790-10797.

[28] H. Kim, K. Lee, S.I. Woo, Y. Jung, Physical Chemistry Chemical Physics 13 (2011) 17505-17510.

[29] G.-L. Chai, Z. Hou, D.-J. Shu, T. Ikeda, K. Terakura, Journal of the American Chemical Society 136 (2014) 13629-13640.

[30] V. Viswanathan, H.A. Hansen, J. Rossmeisl, J.K. Norskov, The Journal of Physical Chemistry Letters 3 (2012) 2948-2951.

[31] T.S. Olson, S. Pylypenko, S. Kattel, P. Atanassov, B. Kiefer, The Journal of Physical Chemistry C 114 (2010) 15190-15195.

[32] L. Chen, G. Wu, E.F. Holby, P. Zelenay, W.-Q. Tao, Q. Kang, Electrochimica Acta 158 (2015) 175-186.

[33] A. Lyalin, A. Nakayama, K. Uosaki, T. Taketsugu, Physical Chemistry Chemical Physics 15 (2013) 2809-2820.

[34] G. Zhang, R. Chenitz, M. Lefevre, S. Sun, J.-P. Dodelet, Nano Energy (present issue).

[35] G. Wu, P. Zelenay, Accounts of Chemical Research 46 (2013) 1878-1889.

[36] L. Wang, A. Ambrosi, M. Pumera, Angewandte Chemie International Edition 52

(2013) 13818-13821.

[37] K. Artyushkova, A. Serov, S. Rojas-Carbonell, P. Atanassov, The Journal of Physical Chemistry C 119 (2015) 25917-25928.

[38] H. Zhu, S.J. Paddison, T.A. Zawodzinski Jr, Electrochimica Acta 101 (2013) 293300.

[39] D.W. Boukhvalov, M.I. Katsnelson, Applied Physics Letters 95 (2009) 023109.

[40] D. Deng, L. Yu, X. Chen, G. Wang, L. Jin, X. Pan, J. Deng, G. Sun, X. Bao, Angewandte Chemie International Edition 52 (2013) 371-375.

[41] Y. Hu, J.O. Jensen, W. Zhang, L.N. Cleemann, W. Xing, N.J. Bjerrum, Q. Li, Angewandte Chemie International Edition 53 (2014) 3675-3679.

[42] K. Strickland, E. Miner, Q. Jia, U. Tylus, N. Ramaswamy, W. Liang, M.-T. Sougrati, F. Jaouen, S. Mukerjee, Nature Communications 6 (2015).

[43] S.H. Noh, D.H. Kwak, M.H. Seo, T. Ohsaka, B. Han, Electrochimica Acta 140 (2014) 225-231.

[44] A. Zitolo, V. Goellner, V. Armel, M.-T. Sougrati, T. Mineva, L. Stievano, E. Fonda, F. Jaouen, Nature Materials 14 (2015) 937-942.

[45] T. Ikeda, M. Boero, S.-F. Huang, K. Terakura, M. Oshima, J.-i. Ozaki, The Journal of Physical Chemistry C 112 (2008) 14706-14709.

[46] Y. Okamoto, Applied Surface Science 256 (2009) 335-341.

[47] M.F. Francis, W.A. Curtin, Nature Communications 6 (2015).

[48] Q. Jia, N. Ramaswamy, H. Hafiz, U. Tylus, K. Strickland, G. Wu, B. Barbiellini, A. Bansil, E.F. Holby, P. Zelenay, S. Mukerjee, ACS Nano 9 (2015) 12496-12505.

[49] K. Artyushkova, B. Kiefer, B. Halevi, A. Knop-Gericke, R. Schlogl, P. Atanassov, Chemical Communications 49 (2013) 2539-2541. 